\title{
STEM-EELS analysis reveals stable high-density He in nanopores of amorphous silicon coatings deposited by magnetron sputtering
}

Roland Schierholz ${ }^{\mathrm{a}, \mathrm{b},{ }^{*}, \text { Bertrand Lacroix }}{ }^{\mathrm{a}}$, Vanda Godinho ${ }^{\mathrm{a}}$, Jaime Caballero-Hernández ${ }^{\mathrm{a}}$, Martial Duchamp ${ }^{c}$ and Asunción Fernández ${ }^{a,{ }^{*}}$

${ }^{a}$ Instituto de Ciencia de Materiales de Sevilla, CSIC-Univ. Sevilla, Avda. Américo Vespucio 49, 41092 Sevilla, Spain

${ }^{\mathrm{b}}$ Institut für Energie- und Klimaforschung, Grundlagen der Elektrochemie (IEK9), Forschungszentrum Jülich GmbH, 52425 Jülich, Germany

${ }^{\mathrm{c}}$ Ernst Ruska-Centre for Microscopy and Spectroscopy with Electrons, Forschungszentrum Jülich GmbH, 52425 Jülich, Germany

* rolandschierholz@gmx.de, asuncion@icmse.csic.es

\section{Abstract}

A broad interest has been showed recently on the study of nanostructuring of thin films and surfaces obtained by low-energy He plasma treatments and He incorporation via magnetron sputtering. In this paper spatially resolved electron energy-loss spectroscopy (EELS) in a scanning transmission electron microscope (STEM) is used to locate and characterize the He state in nanoporous amorphous silicon coatings deposited by magnetron sputtering. A dedicated MATLAB program was developed to quantify the helium density inside individual pores based on the energy position shift or peak intensity of the He K-edge. A good agreement was observed between the high density $\left(\sim 35-60 \mathrm{at} / \mathrm{nm}^{3}\right)$ and pressure $(0.3-1.0 \mathrm{GPa})$ values obtained in nanoscale analysis and the values derived from macroscopic measurements (the composition obtained by proton backscattering spectroscopy coupled to the macroscopic porosity estimated from ellipsometry). This work provides new insights into these novel porous coatings, providing evidence of highdensity He located inside the pores and validating the methodology applied here to characterize the formation of pores filled with the helium process gas during deposition. A similar stabilization of condensed He bubbles has been previously demonstrated by high-energy He ion implantation in metals and is newly demonstrated here using a widely employed methodology, magnetron sputtering, for achieving coatings with a high density of homogeneously distributed pores and He storage capacities as high as 21 at\%.

Keywords: spatially resolved EELS, spectrum imaging, magnetron sputtering, amorphous porous silicon coatings, closed nanopores, condensed He bubbles, reduced refractive index 


\section{Introduction}

The introduction of porosity in nanomaterials has been a topic of increasing interest over the last decade, and research efforts have been focused on the synthesis and characterization of newly designed porous and nanostructured materials. A fundamental understanding of the nanostructure permits the control and design of porous materials with tailored functionalities. Among many others, porous silicon currently remains one of the most actively researched materials for diverse applications such as sensors, photonic devices, microelectronics and solar energy conversion [1-6]. Being fully compatible with the established microelectronics technology, the most attracting feature of porous silicon is the wide range of refractive indexes that can be achieved by varying the percentage of porosity. In a recent work, we proposed a new bottom-up approach to prepare porous silicon coatings by magnetron sputtering as an alternative route to the traditional electrochemical-based processes [7]. In that work, we demonstrated the possibility of producing porous silicon coatings with tailored refractive indices (from 3.75 to 4.75 at $500 \mathrm{~nm}$ ) using magnetron sputtering with $\mathrm{He}$ or Ar as the process gas [7,8]. In addition to the advantages of the well-known industrial scale-up of the magnetron sputtering process, our procedure provides many possibilities for the design and production of various devices alternating for example dense and porous layers, intrinsic or doped silicon, etc. [7]. The low-refractive-index coatings produced exhibit a microstructure of closed pores that can be oriented to the coating's growing direction. In this previous work [7], the incorporation of a high He amount (up to 30 at\%) inside the coatings was detected by proton backscattered spectroscopy, and it was proposed that He was most likely trapped inside the closed pores during deposition [7,8]. In fact, in another previous paper, we used spatially resolved electron energy-loss spectroscopy (EELS) in a scanning transmission electron microscope (STEM) to demonstrate that amorphous porous $\mathrm{SiN}_{\mathrm{x}} \mathrm{O}_{\mathrm{y}}$ coatings can be prepared by magnetron sputtering, revealing closed pores containing molecular $\mathrm{N}_{2}$ inside [9].

At this point of the research, the challenge in the present work is to investigate the location of the $\mathrm{He}$ in the porous $\mathrm{Si}$ coatings as well as to quantify the helium with nano-scale resolution using the STEM/EELS methodology available in the TEM facilities of our laboratory. The incorporation of $\mathrm{He}$ in metals has been well investigated due to its importance in nuclear technology. Ion implantation has been used to re-create the conditions of the reactors mainly to investigate damage caused in materials. In these works, the extremely low solubility of He in metals has been reported to be the cause of the formation of He bubbles during ion implantation [10-12]. In most of these works, helium implanted in crystalline materials leads to the formation at a certain depth of a layer of largely dispersed bubbles with diameters ranging from 2 to $25 \mathrm{~nm}$. 
EELS was used for the evaluation of helium density inside these bubbles. The first approach was to use the blue shift of the He $1 s \rightarrow 2 p$ transition as a function of He density $[10,13]$. Later, spatially resolved EELS was reported to be able to record spectra of single bubbles by several authors $[12,14-16]$. The advantage here is that the helium density can be retrieved at the nanoscale using two different methods. One method uses the integrated intensity under the He K-edge, and the other uses the already mentioned energy shift of the $1 s \rightarrow 2 p$ transition. A description of both methods is given in reference 12 (and the references therein). The first aim of the work presented here was to apply a similar STEM/EELS methodology to confirm that the He incorporated in the amorphous silicon coatings prepared by magnetron sputtering remains inside the closed pores and, if so, to characterize the physical parameters (density, pressure) of He. Both goals were accomplished, as described in this paper, and an ad hoc MATLAB routine was also developed. Additionally, we compare the values for the He density obtained inside the microscope to the values estimated from macroscopic measurements. Note that our results presented in this paper reveal similar stabilization of condensed He in the nanopores (He densities of $\sim 30-50 \mathrm{at} / \mathrm{nm}^{3}$ ) as that previously demonstrated in bubbles formed by high-energy He ion implantation in metals [12,14-16]. A high amount of He can therefore be stored and stabilized at room temperature inside the closed pores. To store a similar density in a macroscopic tank at room temperature, pressures of approximately 1 kbar would be required [17].

To re-create the conditions of the cold plasma in contact with the nuclear reactor walls, some researchers have artificially introduced $\mathrm{He}$ in different metals using also magnetron sputtering in He/Ar mixtures [18-20]. More recently the exposure to He plasmas and He incorporation via magnetron sputtering have also been presented as a route for nanostructuring metals [21-23]. In all these articles, although the presence of He and nanopores was reported by TEM images, no quantification of the He condensed state was provided. At this point, it is important to emphasize the relevance of the present work that newly demonstrates that coatings can be prepared by magnetron sputtering containing a high density of homogeneously distributed pores filled with helium in a condensed state.

\section{Experimental}

\subsection{Deposition details}

In our previous work [7] we showed the dependence of the pore size and distribution on several deposition parameters of the magnetron sputtering deposition. Based on this information, a deposition power of $300 \mathrm{~W}$ in radiofrequency (RF) was selected in this paper to obtain rather 
large and separated pores to facilitate the individual pore analysis by STEM/EELS (see the micrograph shown in figure 1). The magnetron head was placed at a $30^{\circ}$ angle to the normal of the substrate at a distance of $5 \mathrm{~cm}$ (see the inset in figure 1 left). For comparison purposes a dense coating was also deposited using $150 \mathrm{~W}$ in RF-mode and a substrate bias of $100 \mathrm{~V}$. Both, the dense and porous amorphous silicon coatings, were deposited from a pure Si target (Kurt J Lesker 99.999\% pure). Before deposition the base pressure was $10^{-4} \mathrm{~Pa}$. The porous coating was deposited at $\mathrm{p}(\mathrm{He})=4.8 \mathrm{~Pa}$, while the dense coating was deposited at $\mathrm{p}(\mathrm{Ar})=1.3 \mathrm{~Pa}$. Silicon $(100)$ and quartz were used as substrates. Table 1 summarizes the deposition conditions and labels for the studied samples.

\subsection{Characterization of the coatings}

The thickness of the coatings was examined using scanning electron microscopy (HITACHI S4800 SEM-FEG) working at $2 \mathrm{kV}$ on fractured samples without any conductive coating.

The microstructure of the coatings was investigated by transmission electron microscopy (TEM) using a FEI Tecnai F30 microscope operated at 300 kV equipped with a Gatan GIF Quantum 963 energy filter at the Laboratory of Nanoscopies and Spectroscopies (LANE-ICMSE, Sevilla, Spain). Cross-sectional TEM specimens of the coatings deposited on Si substrates were prepared using the conventional approach by mechanical polishing and dimple grinding followed by $\mathrm{Ar}^{+}$ion milling until electron transparency was attained. The quantitative STEM/EELS analysis of individual nano-pores of highly porous coatings requires the preparation of thin lamella of less than $100 \mathrm{~nm}$ thickness, which should include closed filled pores. However, overlap of pores should be avoided, and very small pores will make the STEM/EELS analysis of individual pores more difficult. In this sense the porous sample deposited at $300 \mathrm{~W}$ is very adequate as shown in Fig. 1.

The compositions of the thin films were evaluated using proton backscattering spectrometry (p-BS) at the National Center for Accelerators (CNA, Sevilla, Spain) using a 3-MV tandem accelerator. The spectra were obtained using two different energies, 1.0 and $2.3 \mathrm{MeV}$, for the proton beam and a surface barrier detector set at $165^{\circ}$. To obtain the thickness and composition of the films, both spectra were simultaneously simulated for every sample using the SIMNRA code [24] (see more details in ref. 7)

The optical characterization was performed on coatings on quartz substrates in a UVISEL spectroscopic ellipsometer from HORIBA Jobin Yvon with an incidence angle of $60^{\circ}$ and a wavelength range of $563-1550 \mathrm{~nm}$. The data were analyzed using the Deltapsi2 software developed by HORIBA Jobin Yvon. 


\subsection{STEM-EELS spectrum imaging data analysis}

Annular dark field scanning transmission electron micrographs (ADF-STEM) and spatially resolved electron energy loss-spectra (EELS) were recorded simultaneously in the Tecnai F30 on single pores of various diameters. The spectrum images were acquired in the low-loss region with a pixel size of $1 \mathrm{~nm}$ using subpixel scanning (16*16). At each position, an EELS spectrum of 2048 pixels of length with energy dispersion of $0.05 \mathrm{eV} / \mathrm{channel}$ was recorded using an integration time between 0.05 and $0.07 \mathrm{~s}$.

The He K-edge peak corresponding to the $1 \mathrm{~s}$ to $2 p$ transition was placed at approximately $22 \mathrm{eV}$ on the high energy tail of the Si plasmon at approximately $17 \mathrm{eV}$ [25]. To process the EELS spectrum image dataset and extract the He signal, a MATLAB program was developed based on the method described in reference 12. A version of this program is provided in the supporting information section. First, the raw data were aligned with the zero loss peak (ZLP) and subjected to multivariate statistical analysis (MSA) [26] to remove the statistical noise. The plural scattering distribution was then removed from the spectra using Fourier-log multiple scattering deconvolution (Program FLOG [27]). The specimen thickness map was calculated using the logratio method [27] using the mean free path calculated using the IMFP routine [27]. Using our experimental conditions, a mean free path of $\lambda=145 \mathrm{~nm}$ was obtained using the Malis equation [28]. The contribution of the Si plasmon was fitted with the function given in eq. 1 from reference 29:

$$
I=a E /\left(\left(E^{2}-E_{p}{ }^{2}\right)^{2}+\left(E \Delta E_{p}\right)^{2}\right) \text { (equation 1) }
$$

Here, $E_{p}$ represents the plasmon energy, $\Delta E_{p}$ corresponds to the peak width, and $a$ represents the amplitude. To improve the He signal extraction, a second peak was required at energies higher than $23 \mathrm{eV}$. This contribution may be due to surface oxide $\mathrm{SiO}_{2}$ or carbon contamination (both at approximately $24 \mathrm{eV}$ ). These two plasmon contributions were fitted simultaneously to the data in two ranges (from 16 to $20.5 \mathrm{eV}$ and from 23.5 to $40 \mathrm{eV}$ ) on both sides of the He-K edge. During the fit, the $E_{p}$ value of the second plasmon was fixed between 23 and $25 \mathrm{eV}$. The sum of these two contributions ( $\mathrm{Si}$ and $\mathrm{SiO} 2$ plasmon) is referred to as the background signal for the He-K edge. After subtraction of the background signal, the residual signal was fitted in the He K-edge range from 20.5 to $23.5 \mathrm{eV}$. For the He $1 \mathrm{~s} \rightarrow 2 \mathrm{p}$ transition, a simple Gaussian function was used for fitting as this function was reported to yield good results [12]. An example of the fitting procedure will be shown below in the "Results and Discussion" section (Fig. 3c).

To quantify the atomic density of He at each position of the spectrum image, two methods 
were used. In Method 1 [12], the He density $\mathrm{n}_{\mathrm{He}}$ is given by

$$
\mathrm{n}_{\mathrm{He}}=\frac{\mathrm{I}_{\mathrm{He}}}{\mathrm{I}_{\mathrm{ZLP}}} \frac{1}{\mathrm{~d} \sigma_{\mathrm{He}}} \text { (equation 2) }
$$

where $I_{Z L P}$ and $I_{H e}$ are the integrated intensities of the zero loss peak (ZLP) and the He K-edge, respectively. $\sigma_{\mathrm{He}}$ is the cross-section of the He $1 \mathrm{~s} \rightarrow 2 \mathrm{p}$ transition, which was calculated using the program Sigmak3 [27]. For our experimental conditions (acceleration voltage of $300 \mathrm{kV}$, convergence semi-angle $\alpha=12.4 \mathrm{mrad}$, collection semi-angle $\beta=9.6 \mathrm{mrad}$, and integration range of $3 \mathrm{eV}$ ), we obtained $\sigma_{\mathrm{He}}=6.410^{-24} \mathrm{~m}^{2} . d$ is the pore thickness at the pixel position. This latter value was evaluated as the complement of the specimen thickness at each particular pixel compared with the value at a reference pixel outside the pores. This method was suggested in references 14 and 15 and was cross-checked using the STEM-ADF image contrast assuming a uniform ellipsoidal volume of the pores perpendicular to the growing direction. The results of $I_{H e}$ and $I_{Z L P}$ were stored in 2D maps, and the map of He density was calculated using these parameters.

To confirm these measurements, another method (Method 2) was used for comparison. This method relies on the linear relationship between the blue energy shift $\left(\Delta \mathrm{E}_{\mathrm{He}}\right)$ of the He K-edge compared with its position for the free atom (21.218 eV, ref. 30$)$ and the helium density $\left(\mathrm{n}_{\mathrm{He}}\right)$ :

$$
\Delta \mathrm{E}=\mathrm{C} \times \mathrm{n}_{\mathrm{He}}+\delta \text { (equation } 3 \text { ) }
$$

where $C$ and $\delta$ are constants. The energy shift is attributed to the short-range Pauli repulsion between electrons of the neighboring atoms [13]. In this work, we assume that the He density inside the pores will follow a similar linear law. In the literature, some discrepancies in $\mathrm{C}$ and $\delta$ values can be observed $[10,12,14,15]$, and we used two extreme values of $C$. Method $2(a)$ uses $\Delta E=$ $0.015 n_{\mathrm{He}}+0.45$ [15], and Method 2(b) uses $\Delta E=0.044 n_{\mathrm{He}}+0.07 \mathrm{eV}$ [14]. Finally, the He pressure $p_{\mathrm{He}}$ inside the pores can be estimated from the $\mathrm{n}_{\mathrm{He}}$ values provided by the two methods using a suitable equation of state (EOS) [11,12] depending on the He density range.

\section{Results and discussion}

\subsection{Porous silicon coating with reduced refractive index}

Figure 1 shows the microstructure of the investigated porous coating (a-pSi(300), which consists of a highly porous structure of closed pores oriented in the magnetron direction (Fig. 1a). The high-resolution TEM image (Fig. 1b) shows the shape of the pores in detail and reveals the amorphous structure of the Si-matrix. With a RF power of $300 \mathrm{~W}$ supplied to the magnetron, an increase in pore size is observed compared with the coatings reported in the previous work [7]. 
Pores with diameters ranging from 2 to $60 \mathrm{~nm}$ can be observed for the a-pSi(300) coating. The pores exhibit similar minor diameters as the ones in previous samples [7] (average size of $9 \pm 2$ $\mathrm{nm}$ ), while the major diameters are larger. The pores are easier to see on the ADF-STEM image which presents a higher contrast (Fig. 1c). This image points out that for our EELS analysis (section 3.2), we need to choose a thin region of the specimen to find isolated pores (close to the border) but not too thin so that the pores remain closed.

The proton backscattering ( $p-B S$ ) spectrum of this sample is compared with that of the dense sample in Figure 2. A strong signal corresponding to He inside the coating is observed in the porous sample, corresponding to a He amount of 21 at\% (see Table 2). In addition to Si, the dense sample, a-Si(150)(bias), which was deposited under Ar atmosphere with a voltage applied to the substrate, shows a small signal corresponding to the incorporation of Ar. The incorporation of Ar in samples deposited by magnetron sputtering with an applied substrate bias has been reported previously by other authors [31]; nevertheless, this incorporation does not result in the introduction of porosity (details concerning the microstructure of this dense coating are provided in reference 7).

The introduction of porosity leads to a decrease of both the refractive index and extinction coefficient. Table 3 summarizes the values of the refractive indices and damping constants of both coatings at $605 \mathrm{~nm}$. Using the effective medium approximations [32], one can express the relative electrical permittivity of the porous coating, a heterogeneous media $\left(\varepsilon_{r, \text { eff }}\right)$, as a function of the dielectric constants of the continuous matrix $\left(\varepsilon_{r, c}\right)$ and dispersed phase $\left(\varepsilon_{r, d}\right)$ and the volume fraction $(\phi)$ occupied by the pores (eq. 4 , ref.32):

$$
\varepsilon_{r, \text { eff }}=\varepsilon_{r, c}\left[1-\frac{3 \phi\left(\varepsilon_{r, c}-\varepsilon_{r, d}\right)}{2 \varepsilon_{r, c}+\varepsilon_{r, d}+\phi\left(\varepsilon_{r, c}-\varepsilon_{r, d}\right)}\right] \text { (equation 4) }
$$

We calculated the dielectric constant, $\varepsilon=n^{2}-k^{2}$, using the values of $n$ and $k$ measured by ellipsometry at $605 \mathrm{~nm}$, as listed in Table 3 and derived a porosity of $22 \%$ for the porous coating a$\mathrm{pSi}(300)$ assuming that the dielectric constant of the matrix is similar to that of a-Si(150)(bias) and setting $\varepsilon_{r, d}$ for the pores to 1 [33].

\subsection{Extraction of the He EELS signal and evaluation of physical parameters (density, pressure)}

In Figure 1, it is possible to observe some isolated pores in which the spatially resolved EELS analysis could be performed. Figure 3a presents the ADF-STEM signal acquired in parallel with the spectrum image, which encloses an isolated pore. Therefore, an EELS spectrum corresponds to each one of the $1 \times 1 \mathrm{~nm}^{2}$ pixels in the image. In the ADF signal, the matrix with higher atomic 
number and scattering cross section appears bright, and the pores appear dark. In Figure 3b, three EELS spectra corresponding to three selected pixels at the center, the border and outside of the pore are presented with a vertical shift for comparison purposes.

For the selected pixel $(16,10)$, which corresponds to a zone outside the pore, the low loss spectrum contains a peak corresponding to the Si plasmon at $17 \mathrm{eV}$ [25]. Moving toward the pore, pixel $(5,10)$ at the border, a small peak at approximately $22 \mathrm{eV}$ begins to appear that becomes stronger at the center of the pore, pixel $(6,13)$. This superimposed peak corresponds to the He $\mathrm{K}$ edge [25], confirming the presence of He inside the pores.

To characterize the physical parameters of He in the coating, the signal of the He K-edge at each position was extracted from the EELS spectra following the procedure described in detail in section 2.3 (see also the MATLAB program in the supporting information). To illustrate the method, fits of the background signal for the matrix position outside $(16,10)$ and inside $(6,13)$ the pore are presented in Figures $3 \mathrm{c}$ and $3 \mathrm{~d}$. This background signal includes the peak of the Si plasmon as well as another peak for the surface $\mathrm{SiO}_{2}$ component. The figures also show the residual signal obtained by subtraction of the background and experimental spectra. Note that the He signal at approximately $22 \mathrm{eV}$ is clearly visible in the residual signal inside the pore (Fig. $3 \mathrm{~d}$ ), while a flat line is observed above $16 \mathrm{eV}$ for the position outside the pore (Fig. 3c). These results clearly validate our fitting procedure for the extraction of the He signal. The two additional features in the residual signal at energies of approximately 5 to $10 \mathrm{eV}$ (Fig. 3c) and 10 to $15 \mathrm{eV}$ (Fig. 3d) can be attributed to the $\mathrm{Si} / \mathrm{SiO}_{2}$ interface plasmon [16,34] and the cavity plasmons [16], respectively.

Figure 4 illustrates the methods used to evaluate the He density inside the pores. Using our MATLAB routine (see supporting information) and following the procedures described above, the extracted helium signal was fit with a Gaussian function (Figure 3d), and the corresponding integrals of the He intensity $\left(\mathrm{I}_{\mathrm{He}}\right)$ and energy shift $\left(\Delta \mathrm{E}_{\mathrm{He}}\right)$ relative to the He free atom value were stored in 2D maps (see Figures $4 \mathrm{a}$ and $4 \mathrm{e}$ ). As demonstrated on the elemental intensity map (Figure 4a), helium is located inside the nanopore volume. The local specimen thickness at each pixel position is indicated in Figure $4 \mathrm{~b}$. The pore thickness map (Figure 4c) was prepared by selecting the pixel $(17,9)$ as a reference for the specimen thickness $\left(t_{r e f}=38.8 \mathrm{~nm}\right)$. In this case, a maximum value of $6.5 \mathrm{~nm}$ was observed for the pore thickness, which is comparable to the minor axis of the ellipsoidal pore $(7 \mathrm{~nm})$ measured on the ADF-image in Figure 3a. Using the results of the He intensity and pore thickness, it was possible to prepare the helium density map as described in Method 1 (see eq. 2) and presented in Figure 4(d). By considering only the pixels close to the pore center (to avoid surface effects) [14], the density inside the pore ranges from 25 to 54 at/nm ${ }^{3}$, and 
a mean density of $36 \mathrm{at} / \mathrm{nm}^{3}$ was measured. The decreasing of $\mathrm{n}_{\mathrm{He}}$ from the top the bottom of the pore can be explained by He desorption under electron irradiation, as reported by David et al. $[15,16]$ during STEM recording of the spectrum imaging data.

Following Method 2 (described in section 2.3), Figure 4e shows the local energy shift $\Delta E$ of the He K-edge from the value of the free atoms [30]. Figures $4 \mathrm{f}$ and $4 \mathrm{~g}$ present the corresponding He-density maps calculated according to eq. 3 using Methods 2(a) [15] and 2(b) [14]. By comparing Figures $4 \mathrm{~d}, 4 \mathrm{f}$ and $4 \mathrm{~g}$, a good agreement between the He density obtained using Methods 1 and $2(a)$ is observed.

Table 4 summarizes the $\mathrm{n}_{\mathrm{He}}$ values obtained in two different pores using the above mentioned methods for the characterization of the He state in porous a-Si coatings deposited by magnetron sputtering. For the two pores, a good agreement is observed for the He density using Methods 1 and 2a, which provides a good indication of the reliability of our procedure. Even if different authors agree on the linear character of $\Delta \mathrm{E}$ and $\mathrm{n}_{\mathrm{He}}$ dependence, the values given for $\mathrm{C}$ vary significantly $[10,12,14,15]$. For our density range (approximately $20-70 \mathrm{at} / \mathrm{nm}^{3}$ ), we believe that the system is better described using Method 2a because the law given by David et al. [15] was established for a significant number of recorded data in the $20-100 \mathrm{at} / \mathrm{nm}^{3}$ range. The law given by Taverna et al. [14] (Method 2b) was determined using smaller He densities, 10-40 at $/ \mathrm{nm}^{3}$, and thus does not completely describe our system. This idea is supported by Lucas and co-workers [13], who observed that the $C$ value is also dependent on temperature and density.

Knowing the density of $\mathrm{He}$ inside the pores, the internal pressure becomes a relevant parameter to be determined. At higher atomic densities, the ideal gas law is inaccurate. Walsh [12] discussed different semi-empirical equations of states (EOSs) to calculate the He pressure inside the pores from the measured $\mathrm{n}_{\mathrm{He}}$. For our density range, the Trinkaus EOS [11] is the most suitable. The maximum, minimum and mean He density values described before were used to calculate the He pressure, and the results are presented in Table 4. Using Method 1 and 2(a) for the first pore (Pore\#1, presented in Figs. 3 and 4), a mean pressure of $0.3 \mathrm{GPa}$ was determined, and the pressure locally reaches up to $0.8 \mathrm{GPa}$. In the second pore investigated (Pore\#2), higher mean pressure values of 0.5 and $1 \mathrm{GPa}$ were observed, and the pressure locally reaches up to $2 \mathrm{GPa}$. These differences in the two pores are not necessarily significant considering the large uncertainties of the He density measurements that mainly originate from the difficulty of accurately measuring the local pore thickness and from He desorption during the measurements. Nevertheless, these local measurements provide a good indication that the He pressure inside the pore is in the GPa range, which is several orders of magnitude higher than the pressure in the deposition chamber, $\mathrm{p}_{\mathrm{He}}=4.8$ 
Pa.

Therefore, the obtained values should be taken as representative values of the He state in the coatings. To our knowledge, these results reveal for the first time that He can be stabilized in a condensed state inside the nano-pores of amorphous silicon coatings prepared by magnetron sputtering.

\subsection{Comparison with macroscopic results}

The methodology presented in the previous section is based on the direct nanoscale detection of the He signal in the amorphous silicon coatings. The use of spatially resolved EELS with nanometer-scale resolution coupled with the analysis of the data cube matrix allowed us to measure the He density inside the pores with nanometer resolution. As additional information, we propose here a strategy to determine an average value of the He density inside the pores using macroscopic measurements.

Accordingly, one must first assume a homogeneous distribution of pores in the coating and considering the porosity fraction, $\phi=22 \%$, calculated using the Maxwell-Garnett equation (eq. 4) and presented in Table 3, $1 \mathrm{~nm}^{3}$ of the porous coating corresponds to $0.22 \mathrm{~nm}^{3}$ of the pore volume (and $0.78 \mathrm{~nm}^{3}$ of the dense matrix). Considering the density of dense crystalline silicon, we can calculate a reference value of $50 \mathrm{Si}$ atoms per $\mathrm{nm}^{3}$ for the dense matrix. The total number of $\mathrm{Si}$ atoms in $1 \mathrm{~nm}^{3}$ of porous silicon is therefore $0.78 \times 50=39 \mathrm{Si}$ atoms. Knowing the coating composition as measured by $\mathrm{p}-\mathrm{BS}(79 \% \mathrm{Si}$ and $21 \% \mathrm{He})$, the He amount per $\mathrm{nm}^{3}$ of porous silicon coating is 10 atoms of He. From the results in the previous section, evidence is provided that all the $\mathrm{He}$ is inside the pores; therefore, a He concentration inside the pores of $45.5 \mathrm{at} . / \mathrm{nm}^{3}$ can be calculated. This average value calculated from macroscopic measurements is in good agreement with our mean values obtained at the nanoscale using EELS on different pores (see table 4). The macroscopic value is most likely overestimated because the density of crystalline silicon was assumed for the calculation.

In addition to that the He pressure $(P)$ inside a pore of radius $(R)$ can be estimated as the required value to balance the surface tension $(\gamma)$ of a spherical cavity in a solid [35]:

$$
\mathrm{P}=2 \gamma / \mathrm{R} \text { (equation } 5)
$$

Using the surface energy $\mathrm{\gamma}=1.05 \mathrm{~J} / \mathrm{m}^{2}$ for amorphous Si (given by Hara et al. in ref. 36), and considering a radius $\mathrm{R}$ between 3 and $9 \mathrm{~nm}$ for the pore studied in details in our article (pores are elongated), we obtain an He pressure ranging from about 0.2 to $0.7 \mathrm{GPa}$. This approach gives comparable pressure values than our EELS measurements. 


\section{Conclusions}

Spatially resolved EELS was used here for the first time on a-Si coating with closed porosity formed during deposition by magnetron sputtering, demonstrating the helium trapping inside the pores. A good compromise between the adequate deposition conditions and proper TEM sample preparation (thin enough for analysis without pores overlapping but not too thin to have individual closed pores in the field of view) is relevant for the use of the methodology present in this work.

The MATLAB program developed to extract the He signal proved to be a valuable procedure, and the results of the integrated He-K peak intensity and energy shift were used to calculate the He density, $n_{\mathrm{He}}$, inside the pores in the form of 2D maps. This program is provided in the supporting information section of this article. A good agreement was observed between the results of Method 1 and Method 2a, demonstrating the reliability of the analysis. The He pressure inside the pores was calculated, being very high in the GPa range, while the He working gas pressure during deposition was only a few Pa. A review describing experiments and calculations for density and pressures of helium bubbles stabilized in implanted metals can be found in Ref. 35 . The reported data are consistent to the ones reported here in the coating grown by magnetron sputtering.

A strategy is proposed to provide a rough estimate of the He density in the pores from the bulk proton backscattering measurements coupled to porosity estimations from ellipsometry. The results are on the same order of magnitude as those calculated from spatially resolved EELS measurements.

This study provides new insights on the trapping of gas inside closed porosity formed by magnetron sputtering deposition. Amorphous silicon coatings can be prepared containing a high density of homogeneously distributed pores with He storage capacities as high as 21 at \% at room temperature using a widely used methodology such as magnetron sputtering. In our previous work [7], we also described other experimental deposition parameters that could be used to obtain more than 30 at \% He. Our work newly demonstrates that $\mathrm{He}$ is located inside the nano-pores in a condensed state for coatings prepared by magnetron sputtering. Several applications can be envisaged considering that magnetron sputtering is also one of the most common methods in the vacuum-based deposition coating industry. The tuning of the refraction index was already mentioned as fundamental for optical application devices. The electron excitation of liquid helium produces emission in the vacuum-UV. A possible application of the materials characterized in the present work can be as alternative sources to classical VUV light sources [17]. The extension of the 
method to other materials is currently under investigation in our laboratory.

\section{Acknowledgments}

This work was supported by the EU 7FP (project Al-NanoFunc CT-REGPOT-2011-1-285895 and Integrated Infrastructure Initiative Ref.312483-ESTEEM2); the CSIC (PIE 201060E102, PIE 201460E018); the Spanish Ministry MINECO (projects CSD2008-00023 and CTQ2012-32519); and Junta de Andalucía (TEP217 and PE2012-TEP862). The authors also acknowledge the collaboration with the Ernst Ruska-Centre of the Forschungszentrum Jülich within the ER-C proposal A-084.

\section{Supporting Information}

A version of the program for the STEM-EELS data analysis running with MATLAB is available as supporting information. This program is a user-friendly and semi-automated program that was developed to map the composition of helium confined in small cavities (pores and bubbles) formed in a silicon matrix. For more information, the reader is invited to read the user manual, which is also available in the supporting information. For any publication results from analysis performed using this program, please cite the present article.

\section{Figure captions}

Figure 1- (a) TEM cross-sectional view of sample a-pSi(300). Insets: Deposition geometry and pore orientation schemes. (b) Details of high resolution showing pores shapes. (c) ADF-STEM image of the Si coatings showing the pores in dark.

Figure 2- Proton backscattering spectroscopy of (a) porous sample a-pSi(300) and (b) dense sample a-Si(150)(bias)

Figure 3- (a) STEM-ADF image recorded in the vicinity of one isolated pore. (b) Low loss EELS spectra plotted for the various $(x, y)$ positions indicated in (a) (at the center, at the border and outside the pore). (c) and (d) Fitting analysis of the EELS spectra recorded outside and inside the pore. The green line corresponds to the fit of the main background signal (the dashed lines are the $\mathrm{Si}$ and $\mathrm{SiO}_{2}$ fitted components). The blue curve represents the residual signal containing the He Kedge at approximately $22 \mathrm{eV}$ (only visible inside the pore). The fit of the He K-edge using a Gaussian function is plotted in red (see also details in the inset in (d)).

Figure 4- (a) Helium elemental map (He-K edge integrated intensity $I_{H e}$ map). (b) Specimen thickness map. (c) Pore thickness map. The pixel $(17,9)$, close to the center of the spectrum image was selected as a reference for the specimen thickness $\left(t_{r e f}=38.8 \mathrm{~nm}\right.$ ). (d) He density map $n_{H e}$ 
obtained using Method 1. (e) Energy shift map of the He-K edge, $\Delta \mathrm{E}_{\mathrm{He}}$, relative to the free atom energy $(21.218 \mathrm{eV})$. (f) He density map $\mathrm{n}_{\mathrm{He}}$ obtained using Method 2a. (g) He density map $\mathrm{n}_{\mathrm{He}}$ obtained using Method 2b. To improve the clarity, the pixels outside the pore (of low He-K intensity) have been removed in (d), (e), (f) and (g).

\section{References}

1.- Becker C, Lockau D, Sontheimer T, Schubert-Bischoff $P$, Rudigier-Voigt $E$, Bockmeyer M, Schmidt F and Rech B 2012 Large-area 2D Periodic Crystalline Silicon Nanodome Arrays on Nanoimprinted Glass Exhibiting Photonic Band Structure Effects Nanotechnology 23135302

2.- Jin H and Liu G L 2012 Fabrication and Optical Characterization of Light Trapping Silicon Nanopore and Nanoscrew Devices Nanotechnology 23125202

3.- Zhang K, Seo J H, Zhou W and Ma Z 2012 Fast Flexible Electronics Using Transferrable Silicon Nanomembranes Journal of Physics D: Applied Physics 45143001

4.- Spinelli P, Verschuuren M A and Polman A 2012 Broadband Omnidirectional Antireflection Coating Based on Subwavelength Surface Mie Resonators Nat. Commun. 3692

5.- Qu Y, Liao L, Li Y, Zhang H, Huang Y and Duan X 2009 Electrical Conductive and Optically Active Porous Silicon Nanowires Nano Lett. 94539.

6.- Ge M, Rong J, Fang X, Zhang A, Lu Y and Zhou Ch 2013 Scalable preparation of porous silicon nanoparticles and their application for lithium-ion battery anodes Nano Research 6174

7.- Godinho V, Caballero-Hernández J, Jamon D, Rojas TC, Schierholz R, García-López J, Ferrer F J and Fernández A 2013 A New Bottom-up Methodology to Produce Silicon Layers with a Closed Porosity Nanostructure and Reduced Refractive Index Nanotechnology 24275604

8.- Godinho V, Moskovkin P, Alvarez R, Caballero-Hernandez J, Schierholz R, Bera B, Demarche J, Palmero A, Fernández A and Lucas S 2014 On the Formation of the Porous Structure in Nanostructured a-Si Coatings Deposited by DC Magnetron Sputtering at Oblique Angles Nanotechnology 25355705

9.- Godinho V, Rojas T.C and Fernández A 2012 Magnetron Sputtered a-SiO $\mathrm{N}_{\mathrm{y}}$ Thin Films: A Closed Porous Nanostructure with Controlled Optical and Mechanical Properties Microporous and Mesoporous Materials 149142

10.- Jäger W, Manzke R, Trinkaus H, Crecelius G, Zeller R, Fink J and Bay H L 1982 Density and Pressure of Helium in Small Bubbles in Metals Journal of Nuclear Materials 111-112, 674

11.- Trinkaus, H 1983 Energetics and Formation Kinetics of Helium Bubbles in Metals. Radiat. Effects 78 189-211

12.- Walsh C A, Yuan J, Brown L M 2000 A Procedure for Measuring the Helium Density and Pressure in Nanometre-sized Bubbles in Irradiated Materials using Electron-energy-loss Spectroscopy Philosophical Magazine A 801507

13.- Lucas A A, Vigneron J P, Donnelly S E, Rife JC 1983 Theoretical Interpretation of the Vacuum Ultraviolet Reflectance of Liquid Helium and of the Absorption Spectra of Helium Microbubbles in Aluminum Physical Review B 282485

14.- Taverna D, Kociak M, Stéphan O, Fabre A, Finot E, Décamps B and Colliex C 2008 Probing Physical Properties of Confined Fluids within Individual Nanobubbles Physical Review Letters 100 035301.

15.- David M L, Pailloux F, Mauchamp V and Pizzagalli L 2011 In situ Probing of Helium Desorption from Individual Nanobubbles under Electron Irradiation Applied Physics Letters 98171903. 
16.- David M L, Alix K, Pailloux F, Mauchamp V, Couillard M, Botton G A and Pizzagalli L 2014 In situ Controlled Modification of the Helium Density in Single Helium-filled Nanobubbles Journal of Applied Physics 115123508

17.- Lucas A A, Donnelly S E and Vigneron J P 1983 Vacuum Ultraviolet Spectroscopy of Highpressure Helium Microbubbles in Metals Surface Science 12666

18.- Jia J P, Shi LQ, La, X C, Wang Q F 2007 Preparation of Al Thin Films Charged with Helium by DC Magnetron Sputtering Nuclear Instruments and Methods in Physics Research Section B: Beam Interactions with Materials and Atoms 263446

19.- Shi L, Liu C, Xu S and Zhou Z Y 2005 Helium-charged Titanium Films Deposited by Direct Current Magnetron Sputtering Thin Solid Films 47952

20.- Zheng H, Liu S, Yu H B, Wang L B, Liu C Z and Shi L Q 2005 Introduction of Helium into Metals by Magnetron Sputtering Deposition Method Materials Letters 591071

21.- Tanyeli I, Marot L, van de Sanden M C M and De Temmerman G 2014 Nanostructuring of Iron surfaces by Low-Energy Helium Ions ACS Applied Materials \& Interfaces 635462

22.- Kajita S, Yoshida T, Kitaoka D, Etoh R, Yajima M, Ohno N, Yoshida H, Yoshida N and Tera Y 2013 Helium plasma implantation on metals: Nanostructure formation and visible light photocatalytic response J. Appl. Phys 113134301

23.- Iyyakkunnel S, Marot K, Eren B, Steiner R, Moser L, Mathys D, Düggelin $M$, Chapon $P$ and Meyer E 2014 Morphological Changes of Tungsten Surfaces by Low-Flux Helium Plasma Treatment and Helium Incorporation via Magnetron Sputtering ACS Applied Materials \& Interfaces 611609

24.- Mayer M 1999 SIMNRA, a simulation program for the analysis of NRA, RBS and ERDA, AIP Conference Proceedings 475541

25.- Ahn C C and Krivanek O L 1983 EELS-Atlas Gatan

26.- Bonnet N 1998 Multivariate Statistical Methods for the Analysis of Microscope Image Series: Applications in Materials Science Journal of Microscopy 1902

27.- Egerton R F 2011 Electron Energy Loss Spectroscopy in the Electron Microscope, third edition, Springer Appendix B

28.- Malis T, Cheng S C and Egerton R F 1988 EELS Log-ratio Technique for Specimen-thickness Measurement in the TEM J. Electron Microsc. Tech. 8193

29.- Duchamp M, Boothroyd C B, Moreno M S, van Aken B B, Soppe W J and Dunin-Borkowski R E 2013 Electron Energy-loss spectroscopy of Boron-doped Layers in Amorphous Thin Film Silicon Solar Cells Journal of Applied Physics 113093513

30.- Kuhn H G 1962 Atomic Spectra, London: Longmans p.132.

31.- Williams D S, Baiocchi F A, Beairsto R C, Brown J M, Knoell R V and Murarka S P 1987 Nitrogen, Oxygen, and Argon Incorporation during Reactive Sputter Deposition of Titanium Nitride Journal of Vacuum Science and Technology B 51723

32.- Navid A and Pilon L 2008 Effect of Polarization and Morphology on the Optical Properties of Absorbing Nanoporous Thin Films Thin Solid Films $\mathbf{5 1 6} 4159$

33.- Schmidt J W and Moldover M R 2003 Dielectric Permittivity of Eight Gases Measured with Cross Capacitors International Journal of Thermophysics 24375

34.- Moreau P, Brun N, Walsh C A, Colliex C and Howie A 1997 Relativistic Effects in Electronenergy-loss-spectroscopy Observations of the Si/SiO2 Interface Plasmon Peak Phys. Rev. B 566774

35.- Donnelly S E 1985 The density and Pressure of Helium in Bubbles in Implanted Metals: A Critical Review Radiation Effects 901

36.- Hara S, Izumi S, Kumagai T, Sakai S 2005 Surface energy, stress and structure of well-relaxed amorphous silicon: A combination approach of ab initio and classical molecular dynamics Surface Science 58517 

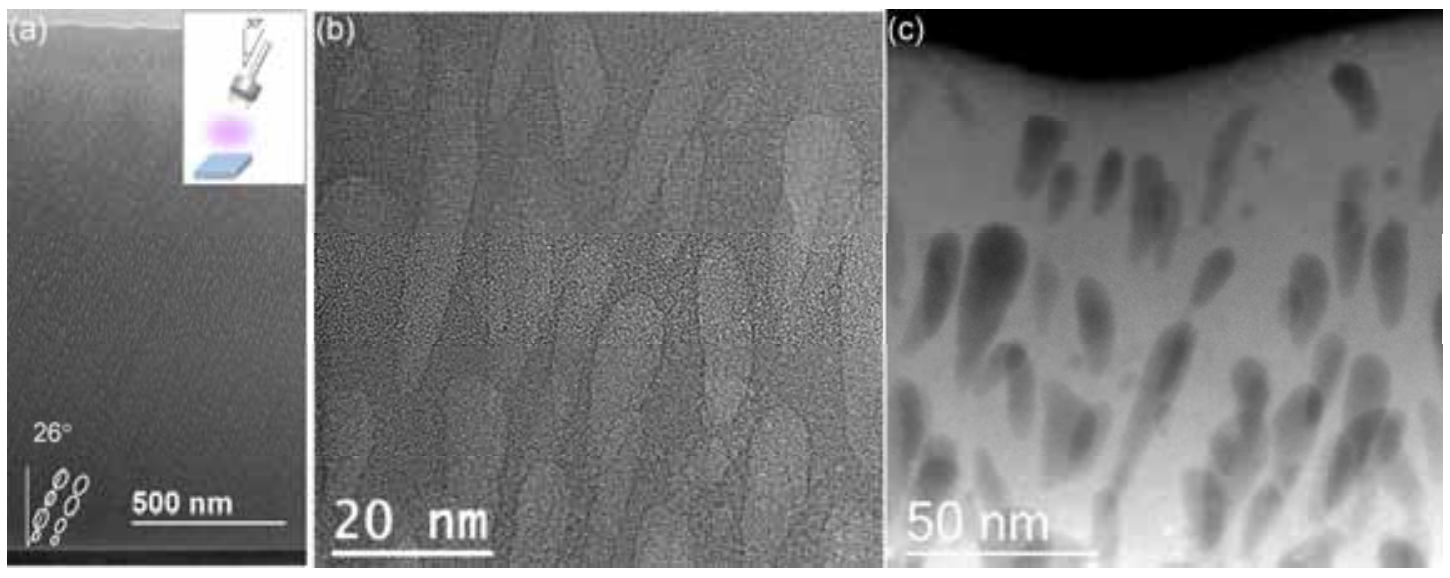

Fig.1

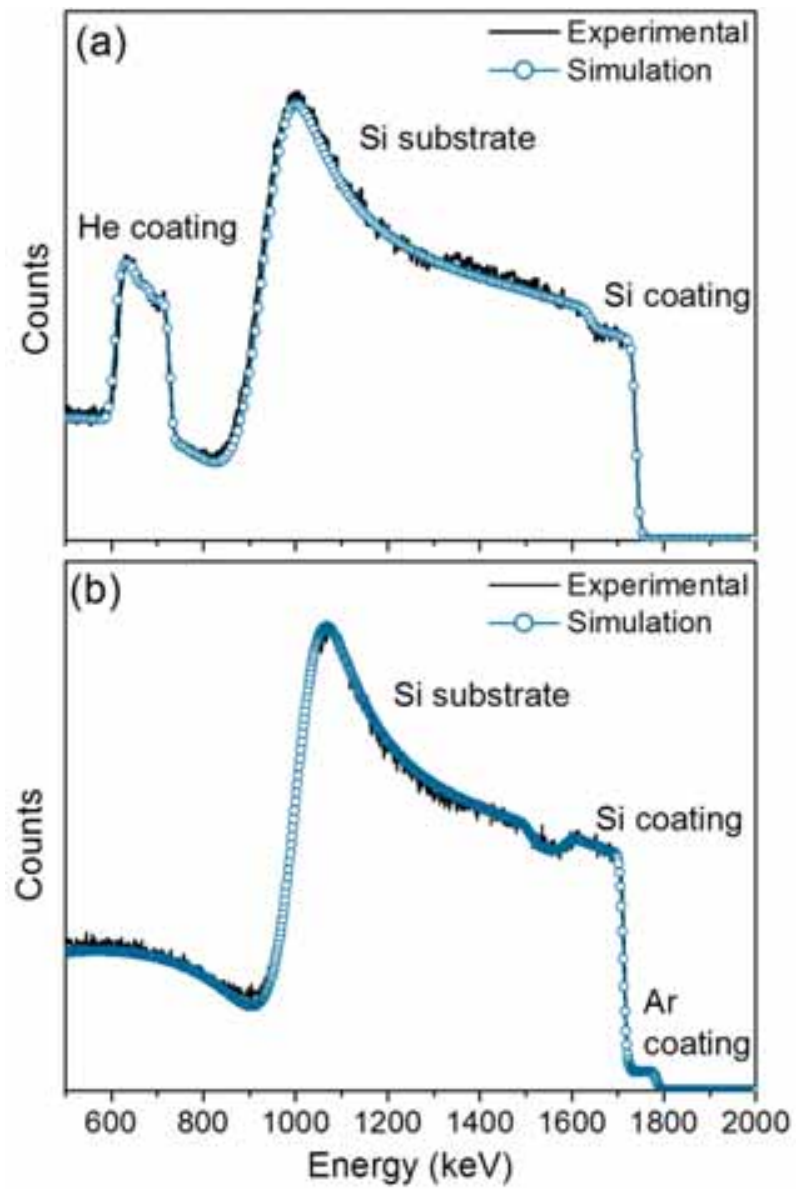

Fig. 2 
(a)<smiles>[X]C[V]</smiles>

\section{$5 \mathrm{~nm}$}

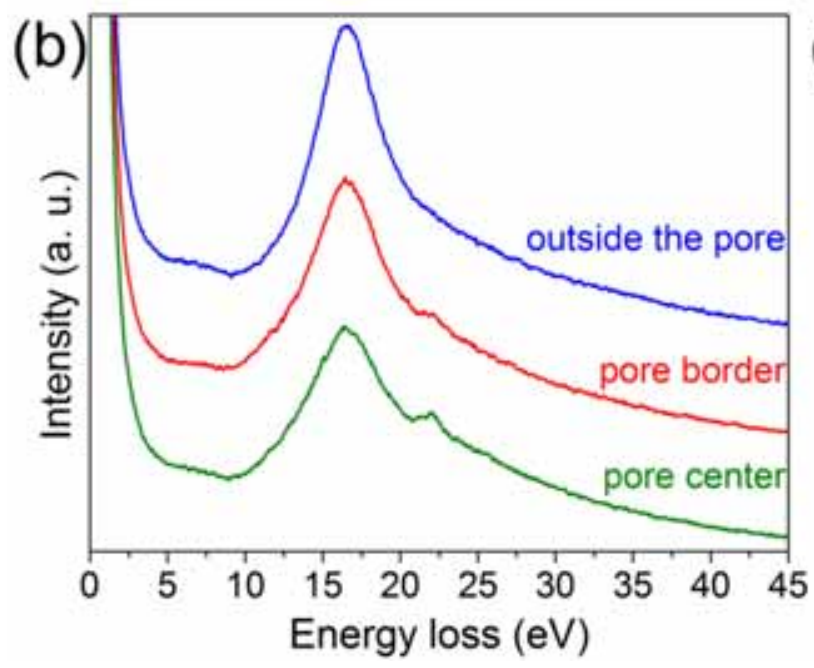

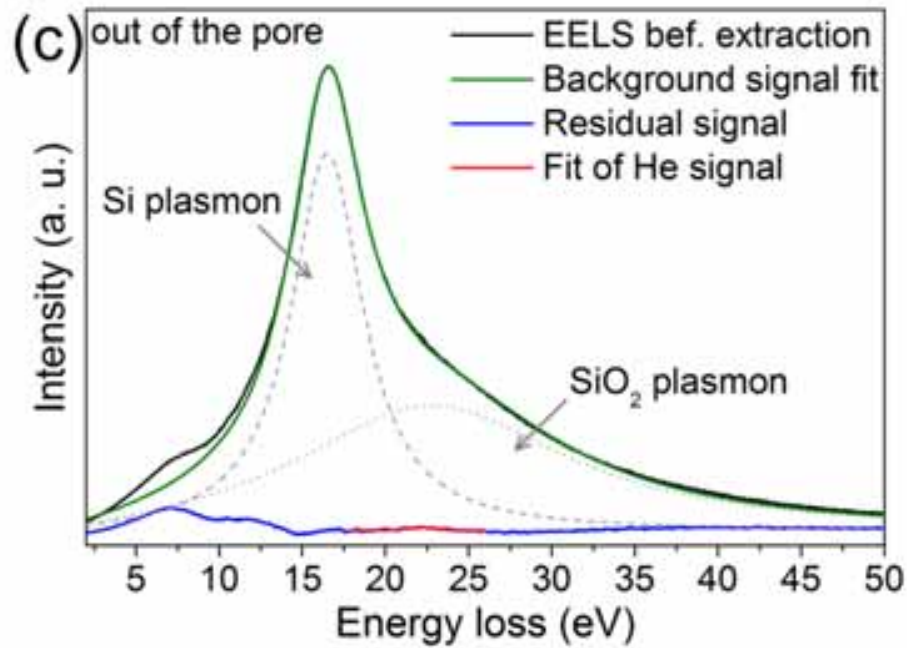

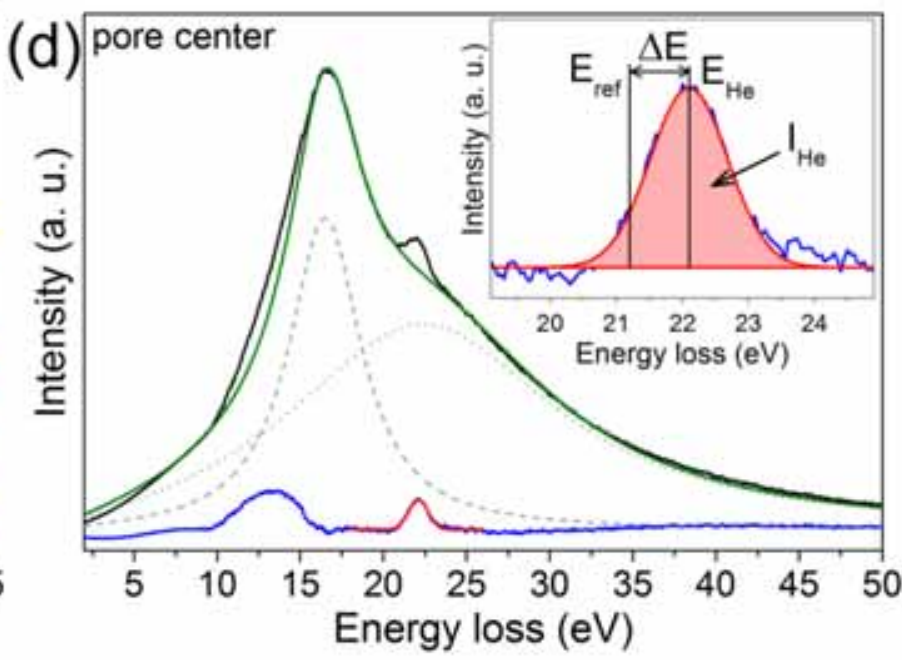

Fig.3 


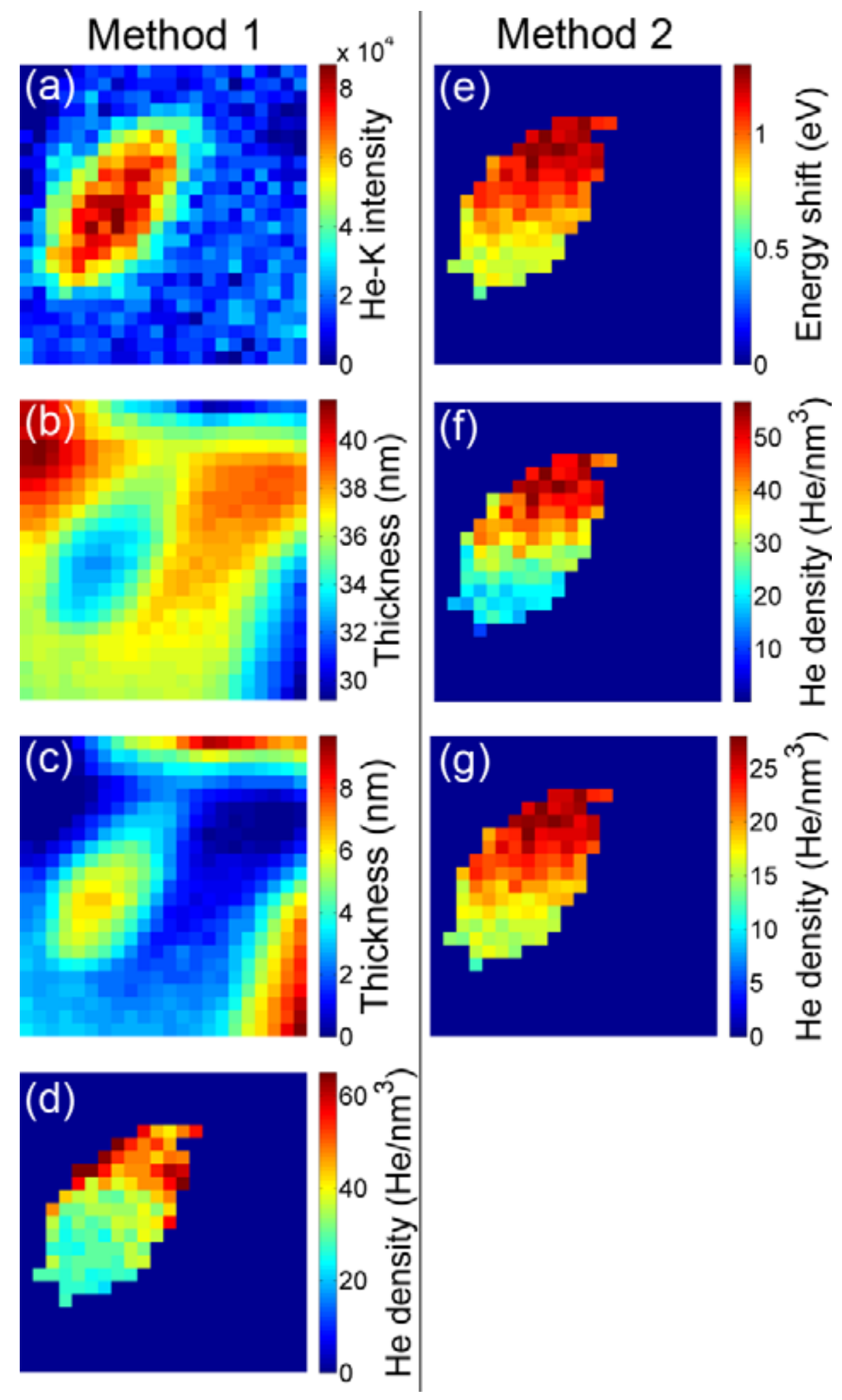

Fig.4 